\title{
Characterizing attentive behavior in intelligent environments
}

\author{
Dalila Durães ${ }^{\mathrm{a}, *}$, Davide Carneiro ${ }^{\mathrm{b}, \mathrm{c}}$, Amparo Jiménez ${ }^{\mathrm{d}}$, Paulo Novais ${ }^{\mathrm{b}}$ \\ a Department of Artificial Intelligence, Technical University of Madrid, Madrid, Spain \\ ${ }^{\mathrm{b}}$ Algoritmi Center, Minho University, Braga, Portugal \\ ' CIICESI, ESTGF, Polytechnic Institute of Porto, Felgueiras, Portugal \\ ${ }^{\mathrm{d}}$ Faculty of Computer Science, Pontifical University of Salamanca, Salamanca, Spain
}

\section{A R T I C L E I N F O}

\section{Article history:}

Received 15 February 2017

Revised 1 May 2017

Accepted 10 May 2017

Available online 17 June 2017

\section{Keywords:}

Ambient intelligent

Machine learning

Learning activities

Attentiveness

Learning styles

\begin{abstract}
A B S T R A C T
Learning styles are strongly connected with learning and when it comes to acquiring new knowledge, attention is one the most important mechanisms. The learner's attention affects learning results and can define the success or failure of a student. When students are carrying out learning activities using new technologies, it is extremely important that the teacher has some feedback from the students' work in order to detect potential learning problems at an early stage and then to choose the appropriate teaching methods. In this paper we present a nonintrusive distributed system for monitoring the attention level in students. It is especially suited for classes working at the computer. The presented system is able to provide real-time information about each student as well as information about the class, and make predictions about the best learning style for a student using an ensemble of neural networks. It can be very useful for teachers to identify potentially distracting events and this system might be very useful to the teacher to implement more suited teaching strategies.
\end{abstract}

(C) 2017 Published by Elsevier B.V.

\section{Introduction}

Education, training, skills development, and learning are processes that are continuously performed since we are born. These characteristics and the ability to learn and teach, allow us to grow up as a person. Besides, learning is inevitably linked to the History of Humanity, to its construction as a social being capable of adapting to new situations. Learning can be defined as the act of acquiring knowledge, behaviors, abilities, standards or preferences and the study of learning has been closely linked to the development of psychology as a science [1].

In a traditional learning environment the student and the teacher are the key elements in the classroom. In environments in which technologies exist, the teacher plays an essential role in providing an engaging learning and teaching environment. Together, teacher and students take a set of physical, social, emotional, mental characteristics, and needs to the classroom. These features influence the way that one relates with the other and consequently affect the way the learning process will progress. Teacher is able to learn about necessities and educational philosophy as well the nature of his/her students; this will improve the teacher's position to facilitate student's learning.

\footnotetext{
* Corresponding author

E-mail addresses: d.alves@alumnos.upm.es (D. Durães),dcarneiro@di.uminho.pt, pjon@di.uminho.pt (D. Carneiro), ajimenezvi@upsa.es (A. Jiménez).
}

For these reasons, the school must create systems that are capable of involving students and capture their attention. Advances in computers and wireless technologies have also had an impact on the educational setting, thus generating a new approach for Ambient Intelligent Systems (AmI systems). The rapid development of these technologies combined with the access to content in a wide variety of settings, allows learners to experience new learning situations beyond the school's walls.

In the last years, the school has introduced computer-based training or networked based learning in order to improve the field of AmI systems [2]. These systems must have the following features: context awareness and context adaptively. The definition of context awareness denotes that the pedagogical progress and the context that are provided to the learning environment should be aware of the situations in which the learner actually is. The context of adaptively refers to the idea that different learning context should be adaptable to the particular setting in which the learner is situated [3]. Using artificial neural network (ANN) it's possible to perform actions for a student and access to information, based on requirements or student profile. In addition, the ANN can be a recommendation system that interacts and represents the student in the system in order to choose relevant items according to their needs and preferences.

Scientific studies have shown the influence of different states of attention on student learning [4-6]. These indicators are useful to predict the behavior of a student and identify potential learning 
problems in the class. By using behavioral biometrics, especially keystroke and mouse dynamics, it is possible to analyze: the type of the task performed by each user; the time spent performing it; as well the level of attentiveness on the task. With this information it is also possible for classifiers to distinguish situations in which each student shows signs of attentiveness and where higher or lower levels of attentiveness are measured [7].

This technique is based only on the observation of the usage of the mouse and the keyboard, which allows an assessment of the student's performance. Consequently, we considered both noninvasive and non-intrusive approaches. In this way we can applicate this technique to develop attention level and management initiatives in the context of learning activities, allowing teachers to perceive student's state of mind and adjust teaching process to the student's needs and behavior.

The target of this paper is to propose architecture of an AmI system aimed at capturing and measuring the level of students' attentiveness in real scenarios and dynamically provide recommendations to the teacher. The students can learn in a relatively anonymous environment without the embarrassment of failure and/or socio-cultural prejudice from personal contact. All the students get the same standardized set of learning materials from the learning activities using technologies in an environment. The student usage of the learning materials can be monitored and in an early stage it's possible to discover if the student dropout and counteractive support can be offered. These environments can be used to give instant feedback from student's level of attention.

\section{Theoretical foundations}

It is crucial to improve learning process and mitigate problems that might occur in an environment with learning technologies. Learning theories provide insights into the very complex processes and factors that influence learning and provide precious information that can be used to design instruction that will produce prime results.

Being a cognitive process, attention is strongly connected with learning [4]. When it comes to acquiring new knowledge, attention can be considered one of the most important mechanisms [5]. The level of learner's attention affects learning results. The lack of attention can define the success of a student. In learning activities, attention is also very important to perform these tasks in an efficient and adequate way.

When students are using technology, and especially when he/she is connected to the Internet, distractions can occur. This happens because they have access to messages from chats, social networks, emails, and other applications like music applications and news sites, which can be more attractive to students. Moreover, these applications can constantly run in the background. When these activities are prolonged for a long period of time they may negatively affect the student's level of attention [8].

\subsection{Attention}

The concept of attention has had different definitions since the nineteenth century, but there is no universally accepted definition of attention. Although, there is a diversity of disciplines that study it. Initially, it was only a study field of psychology. However, in recent years it was object of study in different areas including biology, education science, psychiatry, and computer science [9]. With the existence of these multiple sets of disciplines that study attention, its definition diverges depending on the field of study. For example, we can differentiate the concept of attention in human beings and machines. In humans, attention is processed in the brain while in machines there is a processor unit with a certain memory capacity that will process data. As with the brain, computers should analyze more and more data, but unlike the brain they do not or do rarely, "pay attention" [10] to the data.

Most recently, the concept of attention is commonly used either to describe the active selection of information from the environment or the processing of information from internal sources $[7,11]$.

Attention is the cognitive process of focusing on one aspect of the environment while disregarding others. "Pay attention!" is a phrase repeated by so many teachers all over world [12]. Attention is the first step in the learning process. Students cannot learn, understand, or even remember if they do not listen properly, so, they will fail in the learning process. For almost everyone it is easy to pay attention to subjects or things that are interesting or stimulating to them.

\subsection{Features that influence attention}

Generally, there are some factors that influence the level of attention including stress, mental fatigue, anxiety, emotions, different environment and human health [13].

Stress may have a positive or negative influence. On the one hand it is generally accepted that stressful events increase the level of attention [14-18]. On the other hand, there are cases in which stressful events cause depression or aging [19].

When some activities are prolonged for a long period of time, our brain may feel over headed with such amount of information, and this leads to a potential emergence of mental fatigue, which decreases the level of the students' attention.

A substantial literature shows that anxiety affects perceptual and related processes of attention [20]. Anxiety has an impact on cognition and attention because it is often associated with adverse effects on attention of cognitive tasks [21].

Finally, health problems, mood, and the surrounding environment can also influence the level of attentiveness. Fig. 1 presents a design of factors that influence attention [7].

\subsection{Learning styles}

A learning style is the method that allows an individual to learn best. Different people learn in different ways, each one preferring a different learning style. Everyone has a mix of learning styles, but some people may find that they have a dominant style of learning. Others may find that they have different learning styles in different circumstances. There are several models developed by several authors that try to represent the way people learn [22].

Previous research suggests that, in the context of learning activities, different learning styles can influence learning performance. Learning styles are also considered one of the more important factors influencing learning [23].

Some researchers have argued that learning style is also a suitable indicator of potential learning success because it provides information about individual differences in learning preferences and information-processing [24,25].

Learning style not only specifies how a student learns and likes to learn, but it can also help a teacher to adapt to individual students, so that they might learn successfully. When teacher's methodologies do not support a specific learning style, student will find it more difficult to learn and acquire knowledge. When students are doing learning activities using new technologies, it is extremely important that teacher has feedback from the students' work in order to detect potential learning problems at an early stage so he can choose the appropriate teaching methods.

Learning styles can be defined as cognitive, affective, and physiological features that serve as relatively stable indicators of how learners perceive interaction and respond to their learning environments [26]. 


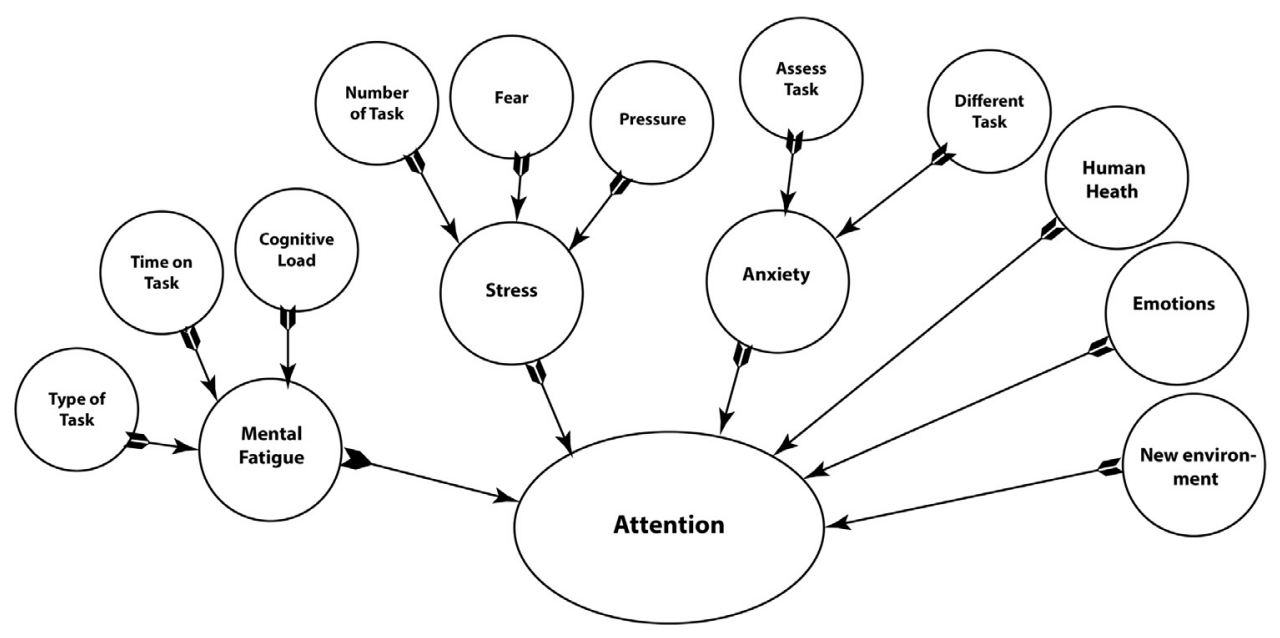

Fig. 1. Conceptualization of the set of factors that influence attention [7].

Table 1

Five dimensions of learning styles [27].

\begin{tabular}{llll}
\hline Definitions & Dimensions & Definitions & \\
\hline Do & Active & Reflexive & Think \\
Learn facts & Sensitive & Intuitive & Learn concepts \\
Needs drawings & Visual & Verbal & Needs read or dissertations \\
Derive facts from facts & Inductive & Deductive & Derive results from principles \\
Step to step & Local & Global & Global framework \\
\hline
\end{tabular}

Learning is improved if the teacher has a system that can detect and classify the learning preferences of students and provide advice from potential learning problems at an early stage in order to choose the most appropriate teaching methods.

Identifying learning styles can be fundamental in order to personalize learning models. Table 1 describes the 5 dimensions of learning styles [27].

In dimensions Active/Reflexive, active experimentation involves doing something with the information, discussing it, explaining it, or testing it in some way. Reflective observation involves examining and manipulating the information introspectively.

In dimensions Sensitive/Intuitive, sensing includes observing and gathering data through the senses. Intuition involves indirect perception by way of the unconscious-speculation, imagination, and hunches.

In dimensions Visual/Verbal, visual learners remember best what they see: pictures, diagrams, flow charts, time lines, films, and demonstrations. Verbal learners remember what they hear and better what they hear and then say.

In dimensions Inductive/Deductive, induction is a reasoning progression that precedes from particulars events (observations, measurements, and data) to generalities (governing rules, laws, and theories). Deduction proceeds in the opposite direction. In induction one infers principles and in opposite, (deduction) one deduces consequences.

In dimensions Local/Global, local learners follow linear reasoning processes when solving problems; global learners make intuitive leaps and may be unable to explain how they came up with solutions.

\subsection{Neural network}

In the last years, the ANN was used to control design techniques and several interesting solutions have been presented for the nonlinear systems $[28,29]$. Another characteristic is the less relatively requiring information of the system dynamics, neural net- works turn out to be a clever move. For this reason, ANN have been proven to be capable of approximating various nonlinear functions to any desired degree of accuracy under certain conditions [30,31].

An ANN can be a recommendation system, which interacts with their student to learn and continually modify the student's profile representing their preferences and choose which items should be relevance to the student. The support for students through ANN can be given in different ways, such as the suggestion of educational resources that fit or adapted their characteristics, which is at the same time the general objective of the recommendation systems.

Some research in learning environments used educational recommender systems to deliver learning objects according to the student's characteristics, preferences and learning needs [32]. In addition, they argue that the development of concrete evaluation frameworks of ANN may focus on incorporating as many evaluation dimensions as possible. These dimensions can address pedagogical dimensions, or on combining a variety of evaluation methods, metrics, and instruments.

There are several data mining algorithms, however for this study we focus on MLP and SVM classifiers.

MLP classifier: Multilayer Perception (MLP) system models are a system designs, used in a large variety of applications. MLP network is one or more layers between input and output layer. Three unique characteristics of MLP are: each neuron in the network includes a nonlinear activation function; hidden neurons are not part of the input or output layer of the network; and the network displays a high degree of connectivity.

SVM classifier: Support Vector Machine is a supervised learning technique for data search, pattern acceptance, and classification based on statistical learning theory. SVM classification creates an $\mathrm{N}$-dimensional hyper plane and separates the data into two categories: Linear SVM and Non-linear SVM. Linear SVM classifier separates the data points used to a linear decision boundary. Non-linear SVM classifier separates the data points used to a non-linear decision boundary.

\section{System approach}

In this section, we provide an overview on the AmI system used for our proposal. This AmI system needs to be trained in order to obtain the learning style of each student. The algorithms used for training the ANN were MLP and SVM classifier. Moreover, the AmI system proposed uses several sources of knowledge to generate recommendations for the students, namely the data collected 


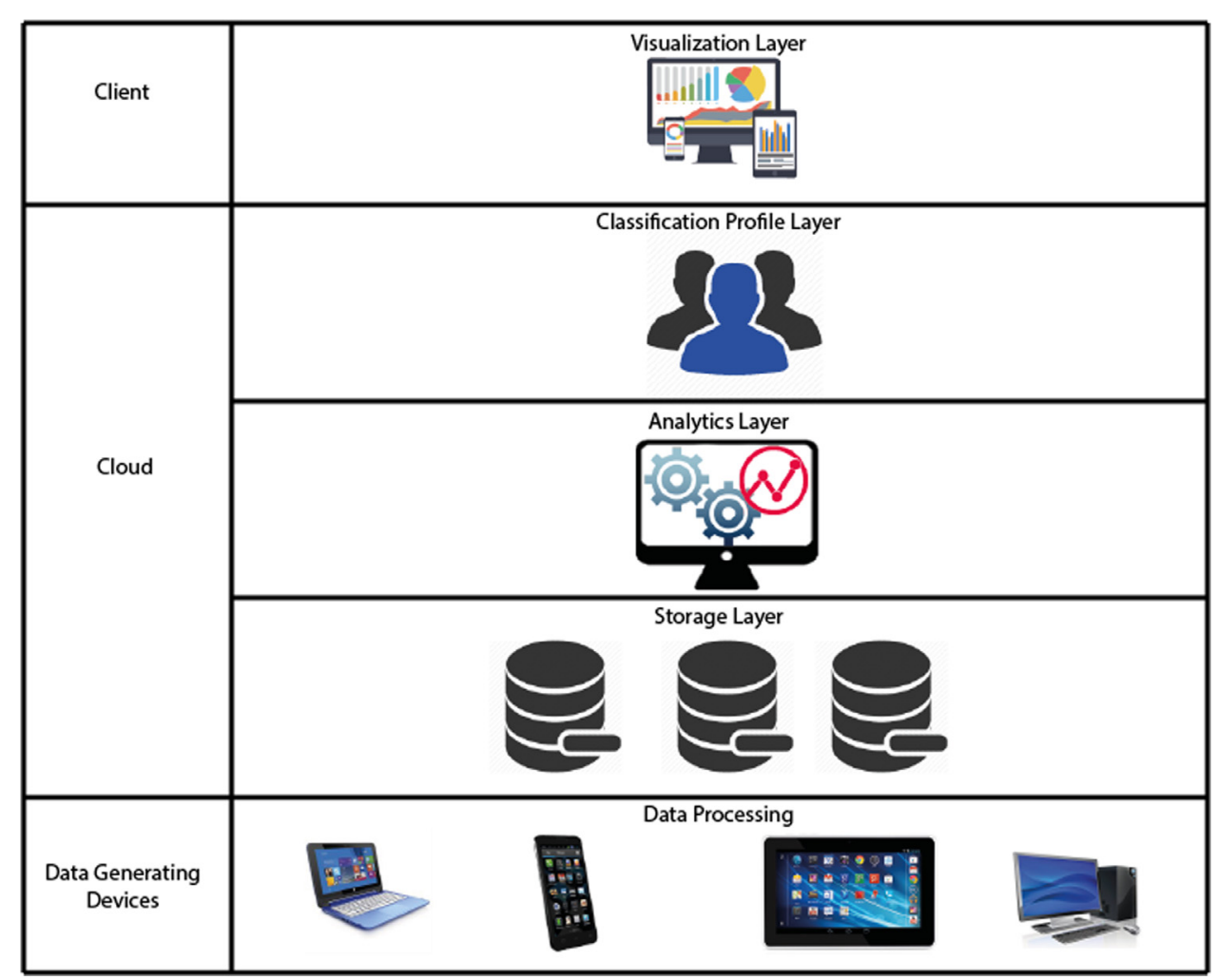

Fig. 2. Architecture of the system.

from the peripheral devices. This data was used for training the system so that he can estimate the learning style of the student's class and recommend.

\subsection{Architecture}

While the student conscientiously interacts with the system and takes his/her decisions and actions, a parallel and transparent process takes place in which the AmI system uses the information. Content and link structures can be adapted to better achieve these goals, although the knowledge level and learning styles better define users.

The system uses three main elements to complete the process: (a) data collected about the student, (b) user model inferred from the collected data, and (c) adaptation tools that advice the teacher.

The architecture of the proposed AmI system, presented in Fig. 2, depicts the process through which the system operates. It is possible to divide it into three major parts: data generating devices, cloud, and client.

The devices where students work have software that generates raw data. These devices store the raw data locally until it is synchronized with the web server in the cloud, which occurs at regular intervals (normally 5 minutes). The cloud is composed of three layers: storage, analytics, and profile classification.

In the storage layer the raw data received from the data generating devices is stored in a data store engine.

The analytic layer provides powerful tools for performing analytics and static analyses in real-time. The system calculates, at regular intervals, an estimation of the general level of performance and attention of each student. This is made by the retrieved data collected in the database and due to the rules defined for the task (by the teacher) calculates the time that each student spent on the task. This layer also contains a ANN that, using the data collected from the peripheral devices, estimates the learning style of each student.
The classification profile layer is where the indicators are interpreted. For example, interpreting data from the attentiveness indicators and building the meta-data, that will support decisionmaking. When the system has a sufficiently large dataset that allows making classifications with precision, it will classify the inputs received into different attention levels in real-time and provide a learning style for each student. This layer has access to the current and historical state of the group from a global perspective, but it can also refer to each student individually, creating each student's learning profile. Profile classification is also a very important aspect to have control of since it allows carrying out analyses within longer time frames. This information will be used by another sub-module, the affective adaptive agent, to provide relevant information to the platform and to the mentioned personalization module.

Finally, the Client layer is developed as a web app with intuitive and visual representation (diagrams and other graphical tools) of the attentiveness states of the group and each student, abstracting from the complexity of the data level where they are positioned. At this point, the system can start to be used by the people involved, especially the instructor, who can better adapt and personalize teaching strategies. With a focus on individual and group performance and using real time analytics, the intuitive visual tools suggest and facilitate decision-making and student management. The actual quantification of the students' attention is displayed in the visualization layer, and can be used to personalize instruction according to the specific student, enabling the instructor to act differently with different students, and also to act differently with the same student, according to his/her past and present level of attention.

\subsection{Methodology}

The aim of this work is to compare a normal and an assessment class at the Secondary School of Caldas das Taipas, Guimarães, 
Table 2

Results of the Kruskall-Wallis test and mean values for each class and each feature.

\begin{tabular}{lllll}
\hline Feature & Symbol & Significance & Mean evaluation & Mean normal \\
\hline Mouse velocity & mv & 0,0011 & 0,49 & 0,53 \\
Mouse acceleration & ma & 0,0010 & 0,54 & 0,57 \\
Click duration & cd & 0,0035 & 245,38 & 159,83 \\
Time between clicks & tbc & 0,0966 & 1964,19 & 3063,11 \\
Distance between click & dbc & 0,0001 & 150,83 & 206,12 \\
Duration distance clicks & ddc & 0,0545 & 143,04 & 143,97 \\
Excess distance between clicks & edbc & 0,0000 & 154,00 & 309,48 \\
Absolute excess distance between click & aedbc & 0,0000 & 1,54 & 2,05 \\
Absolute sum distance between clicks & asdbc & 0,0094 & 4006,31 & 5038,24 \\
Distance point to line between clicks & dplbc & 0,0169 & $21,611,000,00$ & $37,026,200,00$ \\
Absolute distance point between clicks & adpbc & 0,1361 & $157,647,00$ & $208,223,00$ \\
\hline
\end{tabular}

Portugal. We want to determine if classes with different goals have a significant effect on mouse and keyboard dynamics and how can we estimate attention level.

For this purpose, a group of 13 (10 girls and 3 boys) art students were selected to participate, whose average age is seventeen years old. On different weeks, they have a normal and an assessment lesson, where they have access to an individual computer and three hours to complete a task. The lesson started at 8:30 and finished at 11:00 a.m. Students received, at the beginning of the lesson, a document with the goals of the task. The normal and the assessment lessons contained tasks to be completed using Photoshop.

Data collection was carried out using a logger application developed in previous work $[33,34]$. The data collected by the logger application, characterizing the students' interaction patterns, is aggregated in a server to which the logger application connects after the student logs in. This application runs in the background, which makes the data acquisition process, a completely transparent one from the point of view of the student.

\subsection{Data analysis}

In this section we show the existence of different behaviors in the two different lessons. Data was analyzed in two different ways. First, a general analysis was carried out in which statically methods were used to obtain preliminary conclusions. Second, an individual analysis was made in order to compare the different moments.

Although the collected data describes the interaction with both the mouse and the keyboard, only data from the mouse was considered in this analysis. This is due to the characteristics of the task, which was based on Photoshop, that requires mostly the interaction with the mouse The amount of data collected from the keyboard was too small to allow sound analyses. Another important aspect worth mentioning is that Photoshop requires a precise use of the mouse, which makes it a suitable application to the current study.

In a preliminary analysis of the data, we concluded that there are indeed different interaction patterns depending on the type of lesson analyzed. To conclude this, we considered the distributions of the data collected and analyzed the statistical significance of their differences. To this end, we used the Kruskal-Wallis test. Table 2 details the mean value of each feature in each class (evaluation and normal class). It also details the $p$-value of the KruskalWallis test.

When data from the two classes is compared, the first conclusion is that the differences observed are statistically significant in nearly all features, with the exception of Time Between Clicks, Distance During Clicks, and Average Distance Point Between Clicks.

Moreover, the mean values of the features are consistently lower in the evaluation class. In most of the features, this indicates an increased performance (e.g. a smaller average distance between clicks means that the student moved the mouse in a more effi- cient manner). However, in the case of mouse velocity, for example, a smaller velocity could point out a slower, and thus less efficient, movement. In past work we concluded that a slower mouse velocity is indeed necessary for the student to achieve increased accuracy in mouse movement: moving the mouse too fast would make precise movements more difficult to carry out. This is especially true in tasks such as those of this study. A similar trend happens with mouse acceleration and click duration. The remaining features consistently show increased performance in the evaluation class.

Fig. 3 shows the distribution of the values for all the users and for each feature. It is clear that the results of each feature are very different in the two lessons. The students react in different ways in the assessment and normal lesson. We can also consider that, in general, they are more focused on the assessment lesson because they have a slower mouse velocity and mouse acceleration, a smaller distance of the point to line between clicks and higher click duration.

The selected features characterize several aspects of interaction on both normal and assessment lessons. However, this doesn't mean that they are all affected equally or that they are all affected at all when they have an assessment. A curious property of these features comparing the normal and the assessment lesson was that the mouse velocity and the mouse acceleration are lower and the click duration is higher in the assessment lesson. Fig. 4 compares the histograms of $\mathrm{mv}$, ma, and cd and evidences the differences between the two classes.

From these studies we conclude that the most significantly affected features are mv, ma, cd, tbc, dbc, ddc, edbc, ssdbc, and dplbc. In all these features almost all students show statistically significant differences when comparing the two lessons.

\subsection{Class prediction}

One of the aspects we would like to evaluate in this study is the influence of the class in the student, taking into account the learning techniques and all the surroundings in the environment. In this sense, we apply neural networks to classify the classes and estimate a class from the results obtained from a student. After studying the various classification techniques applied in learning systems, this part discusses an ensemble method proposed that combines the predicted outputs from two different classifiers, namely MLP, the traditional classifier and SVM, to obtain better classification results.

The data is supplied to the MLP and SVM classifiers respectively and then the results from both the classifications are combined to give an improved prediction of the data classification.

Initially, we proposed an approach based on combining the outputs of two or more experts (MLP and SVM). This solution can be appropriated in some cases. However, we observed that the simple combination of the experts does not provide learning and 
mv

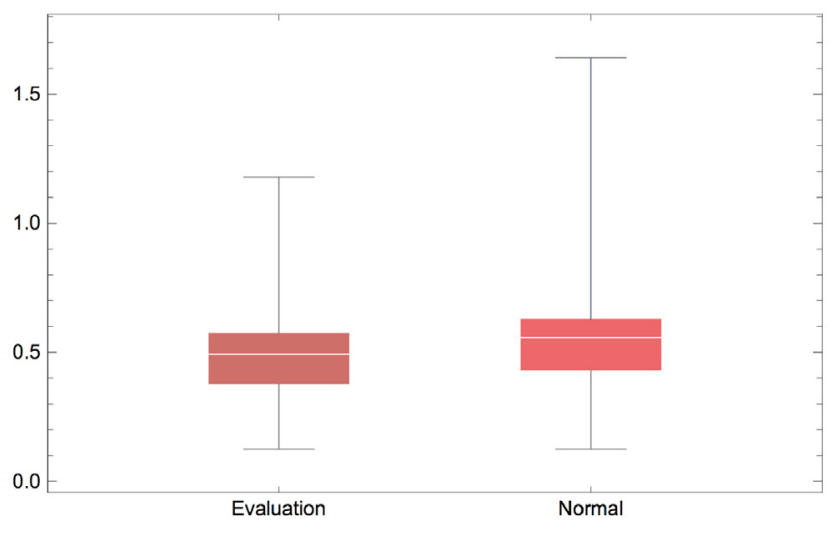

cd

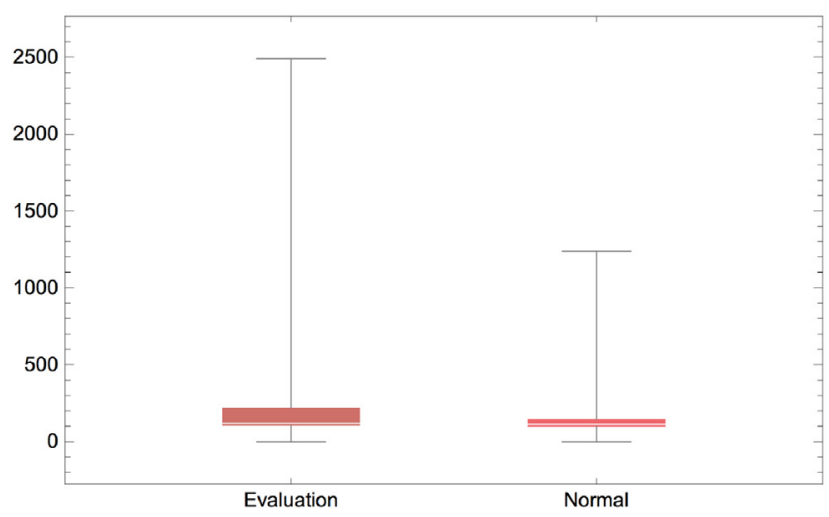

ma
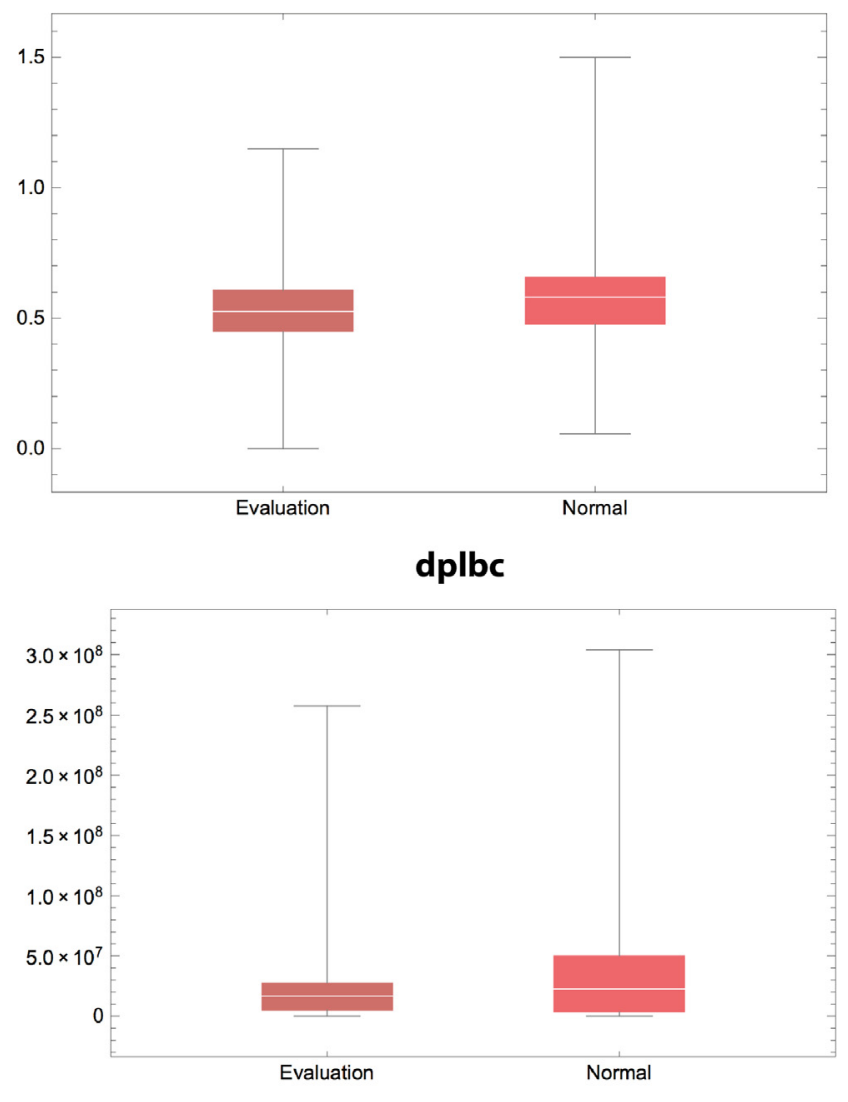

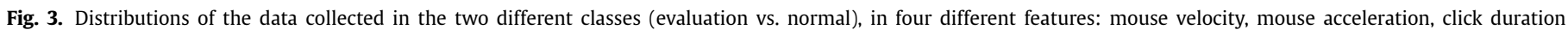
and distance of the pointer do the line between clicks.
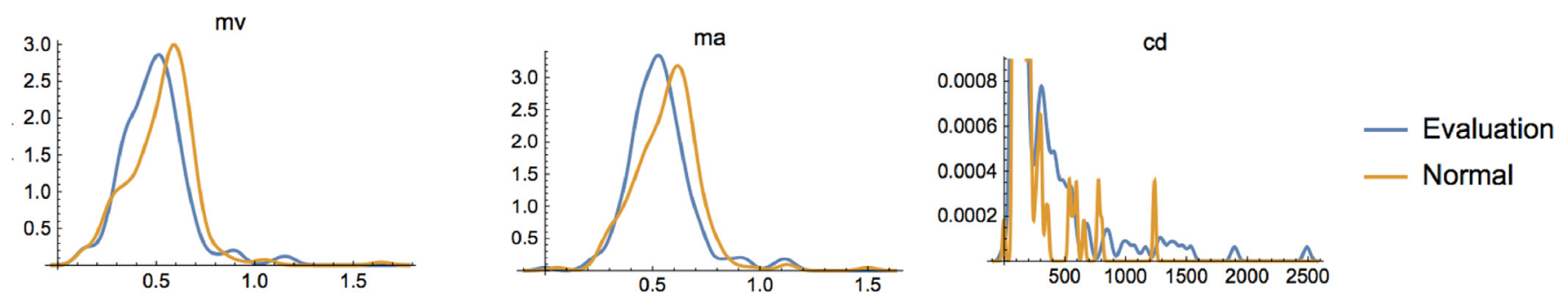

Fig. 4. Comparing mv, ma, and cd during the lessons.

adaptation capacities, which makes it difficult to improve the output of the individual experts. Thus, we proposed the solution shown in Fig. 5 where the outputs of the individual experts are combined taking into account the input data. This way, it is possible to take into account both the characteristics of the input data and the strategies of the individual classifiers and to obtain an automated learning model. In our proposal, we have implemented two different strategies for the ensemble of information: MLP and SVM. Both of them are analyzed in detail in the experiments.

We have implemented a hybrid ANN in order to predict the learning style of the students, which the system received the data from biometrics behavior and used tasks. It's a hybrid prediction method based on MLP and SVM classifier that uses these sources of knowledge and provide the predictions of learning styles for which the system is able to generate more arguments to justify their suitability. Fig. 6 presented this system where the same information is the input on MLP classifier, SVM classifier. The output class provide a predict learning style of each student.

\section{Preliminary results}

During the two lessons, the monitoring system was used to assess the interaction of the students with the computer and to quantify their level of attentiveness. To quantify attentiveness the following methodology was followed. Asides from capturing the interaction of the students with the computer, the monitoring system also registers the applications with which students are interacting. We analyzed all the applications used by all students and labeled each one of them as belonging to the task or not. We then quantify the amount of time that each student spends interacting with applications related to the task versus other applications.

Table 3 details the results of this quantification. Students have a clearly different attitude in the two lessons: when being evaluated, they spend more time interacting with task-related applications, a sign that they are more focused on the task. The results thus point out, not only, that it is possible to quantify student attentiveness in a non-intrusive way, but also that attention is higher when 


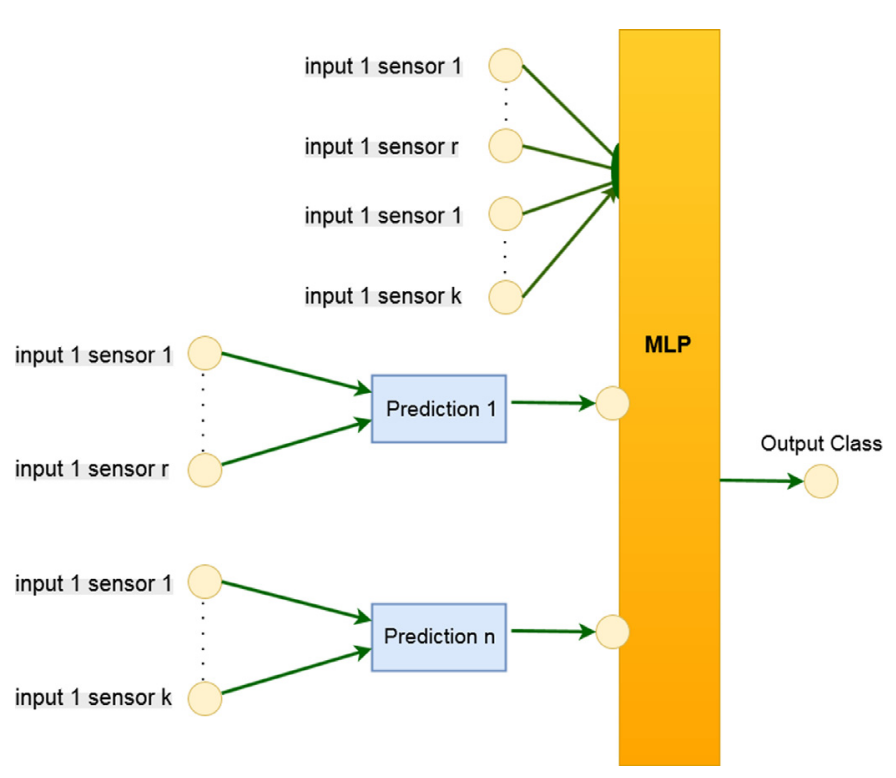

Fig. 5. An ensemble for classification. Ensemble composed of the input data and the output of individual experts.

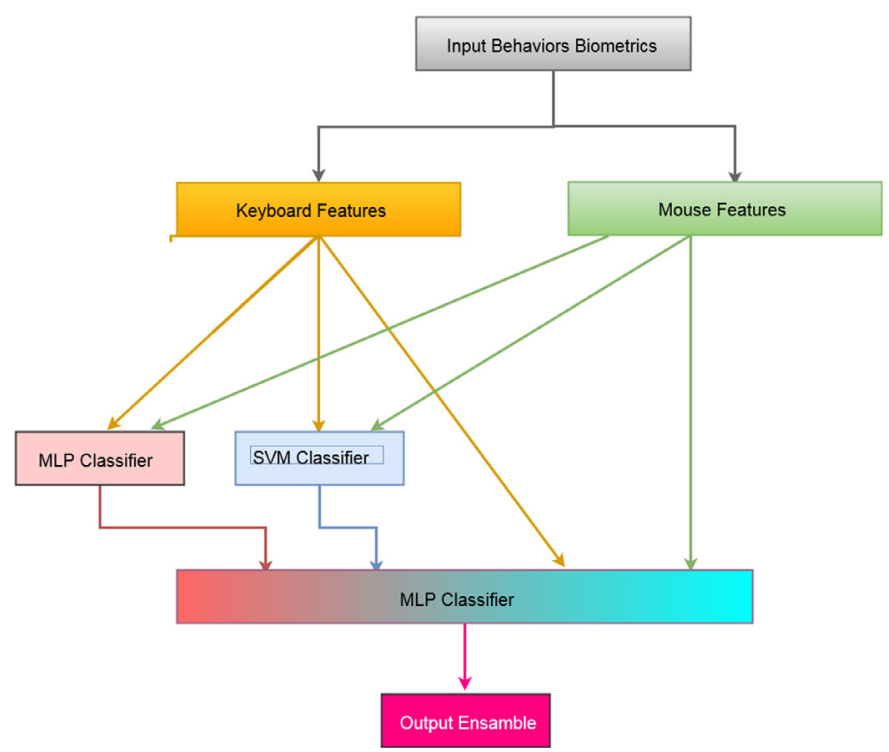

Fig. 6. Ensemble composed of the input data, SVM and MLP classifier, and the output of individual experts.

Table 3

Total time (seconds) devoted to the task and percentage of total time devoted to the task, while being assessed and while in a normal class, for each student.

\begin{tabular}{llllll}
\hline \multirow{2}{*}{ Student } & \multicolumn{2}{l}{ Assessment } & & \multicolumn{2}{l}{ Normal } \\
\cline { 2 - 3 } \cline { 5 - 6 } & Total time $(\mathrm{s})$ & \%Time (\%) & & Total time $(\mathrm{s})$ & \%Time (\%) \\
\hline T7110001 & 315,622 & 67 & 69,020 & 31 \\
T7110003 & 241,601 & 55 & 125,307 & 32 \\
T7110005 & 247,514 & 63 & 180,726 & 40 \\
T7110006 & 157,111 & 36 & 49,407 & 19 \\
T7110007 & 317,728 & 53 & 70,126 & 30 \\
T7110008 & 249,251 & 58 & 78333 & 26 \\
T7110009 & 426,473 & 72 & 213,049 & 53 \\
T7110010 & 323,952 & 74 & 145,113 & 40 \\
T7110011 & 384,571 & 71 & 73,786 & 20 \\
T7110012 & 358,157 & 58 & 15,773 & 30 \\
T7110013 & 83,524 & 48 & 158,886 & 40 \\
\hline
\end{tabular}

Table 4

Classification for keyboard, mouse and tasks.

\begin{tabular}{llll}
\hline & MLP (\%) & SVM (\%) & Ensamble (\%) \\
\hline Keyboard & 72 & 81 & 84 \\
Mouse & 65 & 63 & 69 \\
Tasks & 83 & 84 & 86 \\
\hline
\end{tabular}

under evaluation. This was an expected conclusion but it nonetheless validates the proposed approach.

We also analyzed the correlation between the level of attentiveness and the score of the students in the task. There is a weak positive correlation $(0.41)$ between the two variables. We believe that this value is not higher for two reasons: (1) this class is mostly composed by "excellent" students and (2) the task was of average difficulty. Thus, there were not many differences in the scores. In future work we will study this relationship in more detail, namely in different classes and with tasks of different levels of difficulty.

It is imperative to mention that these features aim to quantify the student's work and estimate the level of attentiveness. These experiments allow drawing some interesting conclusions about students and their behavior during the assessment. However, this test was implemented in a good class with higher scores in all subjects. Yet, they react in different ways during normal or assessment lessons. We can see that they are more focused on the assessment lesson so they have a higher attention level in these situations. The teacher needs to improve the results the level of attention in normal class because he has an average of $33 \%$ of student work. This application shows to the teacher that he needs to change the activity; he must improve different ways how he presents the activity or the level of difficulty in order to increases attentiveness.

We also concluded that there are also significant differences in the interaction patterns when comparing the two lessons, where the students react in different way in a normal and assessment lesson.

The classification tests for the keyboard, mouse and tasks data were carried out splitting the data into training (70\%) and test (30\%) datasets using the Resample filter for each type of lesson detected in each experiment. The training datasets from all the classes are then combined to form the final training dataset. The results obtained are (shown in Table 4.)

It is necessary to remark that the results obtained demonstrate that it is very difficult to predict the class from the data obtained.

\section{Discussion and conclusions}

The work developed so far resulted in a very useful system for the teachers that can improve learning strategies in order to increase learning of his/her students. However, the data collected from each student was very limited. As mentioned in Section 2.3, each student has his/her learning styles and reacts differently in different days of the week and even in different times of the day. For this reason, this system must collect a certain amount of data of each student in different hours of the day and in different days of the week. With this data collected, the system will create a profile for each student in the class that is accessible to the teacher.

When the system has dataset that are large enough it may perform a more precise classification, classifying the inputs received into different attention levels in real-time. Using this, the teacher may take different measures depending on the profile of the group and/or profile of each student.

The Classification layer has access to the current and historical state of the group from a global perspective, but can also refer to each student individually. For that, this layer uses the machine learning mechanisms. After the classification, the enhancing user behavior profile is actualized in the Database Behavior Profile. 
Technology makes enhanced learning/teaching processes possible, overcoming restrictions such as qualified instructor's availability, time restrictions, and individual monitoring, just to name a few. A framework was proposed to address these issues, especially to monitoring students in learning activities. Narrowing the scope of the study, a model to detect attentiveness was proposed, through the use of a developed log tool. With this tool it is possible to detect those factors dynamically and non-intrusively, making it possible to foresee negative situations, allowing to take actions to mitigate them.

The door is thus open to intelligent platforms that allow to analyze students' profiles, taking into account their individual characteristics, and to propose new strategies and actions. By providing instructors with access to this information, we allow them to better manage their interactions with the students, namely by pointing out the most problematic cases of inattention in real-time.

\section{Acknowledgments}

This work has been supported by COMPETE: POCI-01-0145FEDER-007043 and FCT - Fundação para a Ciência e Tecnologia within the Project Scope:UID/CEC/00319/2013.

\section{References}

[1] J.M. Carroll, Human-computer interaction: psychology as a science of design, Annual Rev. Psychol. 48 (1997) 61-83, doi:10.1146/annurev.psych.48.1.61.

[2] W.B. Neto, F. Gauthier, S.M. Nassar, An adaptive e-Learning model for the semantic web, in: Proceedings of the International Workshop on Applications of Semantic Web Technologies for E-Learning, Banff, Canada, 2005, pp. 63-64.

[3] M. Milrad, L.H. Wong, M. Sharples, G.-J. Hwang, C.-K. Looi, H. Ogata, Seamless learning: an international perspective on next generation technology enhanced learning, in: Z.L. Berge, L.Y. Muilenburg (Eds.), Handbook of Mobile Learning, Routledge, New York, 2013, pp. 95-108.

[4] A. Pimenta, S. Gonçalves, D. Carneiro, F. Riverola, P. Novais, Mental workload management as a tool in e-learning scenarios, in: C. B-Peces, O. Paillet, A. Ahrens (Eds.), Proceedings of the 5th International Conference On Pervasive and Embedded Computing and Communication Systems, Scitepress, 2015, pp. 25-32, doi:10.5220/0005237700250032.

[5] K. Hwang, C. Yang, Automated inattention and fatigue detection system in distance education for elementary school students, J. Edu. Technol. 12 (2009) 22-35.

[6] M.W. Esysench, Anxiety: The cognitive Perspective, Erlbaum, Hove, England, 1992

[7] D. Durães, D. Carneiro, J. Bajo, P. Novais, Using computer peripheral devices to measure attentiveness, Advances in Intelligent System and Computing, 2016, pp. 147-155, doi:10.1007/978-3-319-40159-1_12.

[8] A. Pimenta, D. Carneiro, J. Neves, P. Novais, A neural network to classify fatigue from human-computer interaction, Neurocomputing 172 (2015) 413-426, doi:10.1016/j.neucom.2015.03.105.

[9] M. Mancas, Computational Attention - Toward Attentive Computers, Press Universitaire de Louvain, 2007.

[10] W. James, Principles of Psychology, 1, Dover Publications Inc., New York, 1890, pp. 255-260.

[11] B.A. Campbell, H. Hayne, R. Richardson, Attention and information processing in infants and adults, Perspectives from Human and Animal Research, Psichology Press, New York and London, 1992.

[12] R. Pekrun, Emotions and Learning, Educational and Practices - 24, International Academy of Education and International Bureau of Education, United Nations Educational Scientific, 2014, pp. 8-10.

[13] A. Pimenta, D. Carneiro, P. Novais, J. Neves, Monitoring mental fatigue through the analysis of keyboard and mouse interaction patterns, Hybrid Artificial Intelligent Systems, Springer, Berlin Heidelberg, 2013, pp. 222-231, doi:10.1007/ 978-3-642-40846-5_23.

[14] M. Olff, et al., The psychobiology of PTSD: Coping with Trauma, Psichoneuroendocrinology 30 (2005) 974-9 82

[15] M.S. Oitzl, E.R. Kloet, Selective corticosteroid sntagonists modulate specific aspects of spatial orientation learning, Behav. Neurosci 106 (1992) 62-71.

[16] C. Sandi, S.P. Rose, Corticosterone enhances log-term retention in one-day-old chicks trained in a weak passive avoidance learning paradigm, Brain Res. 647 (1994) 106-112.

[17] C. Sandi, Experience-dependence facilitating effect of corticosterone on spatial memory formation in the water maze, Eur. J. Neurosci. 9 (1997) 637-642.

[18] B. Roosendaal, J.L. MacGaugh, Amygdaloid nuclei lesions differentially affect glucorticoid-induced memory enhancement in a inhibitory avoidance, Task. Neurobiol. Learn. Mem. 65 (1996) 1-8.
[19] T.J. Shors, Stressfull experience and learning across lifespan, Annu. Rev. Psychol. 57 (2006) 55-85, doi:10.1146/annurev.psych.57.102904.190205.

[20] J.L. McGaugh, The amygdala modulates the consolidation of memories of emotionally arousing experiences, Annu. Rev. Neurosci. 27 (2004) 1-28, doi:10. 1146/annurev.neuro.27.070203.144157

[21] S. Bishop, Trait anxiety and impoverished prefrontal control of attention, Nature Neurosci. 12 (2008) 92-98, doi:10.1038/nn.2242.

[22] R. Morgan, F. Baker, U.S. Alabama, A Comparison of VARK analysis studies and recommendations for teacher educators, in: Proceedings of the Society for Information Technology \& Teacher Education International Conference, Louisiana, US, 2013, pp. 1381-1385.

[23] N. Ford, Y. Chen, Individual differences, hypermedia navigation and learning: an empirical study, J. Educ. Multim. Hypermedia 9 (4) (2000) 281-311.

[24] D. Kolb, Experiential Learning: Experience as the Source of Learning and Development, Prentice-Hall Inc, New Jersey, 1984.

[25] P.L. Smith, T.J. Ragan, Instructional Design, Wiley, New York, NY, 1999.

[26] J.W. Keefe, Learning style: an overview, NASSP's Student Learning Styles: Diagnosing and Prescribing Programs, 1979, pp. 1-17.

[27] W. Weber, J.M. Rabaey, E. Aarts, R.M. Felder, L.K. Silverman, Learning and teaching styles in engineering education, J. Eng. Educ. 78 (7) (1988) 674-681.

[28] C.L.P. Chen, G.-X. Wen, Y.-J. Liu, F.-Y. Wang, Adaptive Consensus control for a class of nonlinear multiagent time-delay systems using neural networks, IEEE Trans. Neural Netw. Learn. Syst. 25 (6) (2014) 1217-1226.

[29] B. Xu, C. Yang, Z. Shi, Reinforcement learning output feedback nn control using deterministic learning technique, IEEE Trans. Neural Netw. Learn. Syst. 25 (3) (2014) 635-641.

[30] A.U. Levin, K.S. Narendra, Control of nonlinear dynamical systems using neural networks. II. Observability, identification, and control, IEEE Trans. Neural Netw. 7 (1) (1996) 30-42

[31] S.S. Ge, C.C. Hang, T. Zhang, Adaptive neural network control of nonlinear systems by state and output feedback, IEEE Trans. Syst., Man, Cybern. B, Cybern. 29 (6) (1999) 818-828.

[32] P. Rodriguez, S. Heras, J. Palanca, D. Nestor, V. Julian, Argumentation-based hybrid recommender system for recommending learning objects agreement technologies. EUMAS 2015, AT 2015: Multi-Agent Systems and Agreement Technologies. Vol. 9571, 2015, pp. 234-248. doi: 10.1007/978-3-319-33509-4_19.

[33] D. Durães, A. Jiménez, J. Bajo, P. Novais, Monitoring level attention approach in learning activities, Adv. Intel. Syst. Comput. 478 (2016) 33-40, doi:10.1007/ 978-3-319-40165-2_4.

[34] D. Carneiro, A. Pimenta, J. Neves, P. Novais, A multi-modal architecture for non-intrusive analysis of performance in the workplace, Neurocomputing 231 (2017) 41-46, doi:10.1016/j.neucom.2016.05.105

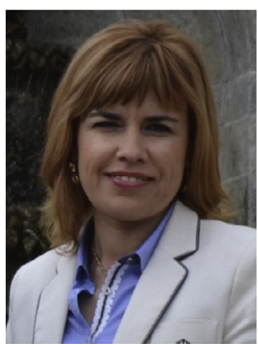

Dalila Durães is a young researcher at the Department of Artificial Intelligence in Technical University of Madrid, Spain. She is Ph.D. student that collaborate with ISLab (Intelligent System Lab) at Department of Informatics, University of Minho, Braga, Portugal. She develops scientific research in the field of Artificial Intelligence, with applications in Human-Computer Interaction and Learning Frequent Behavioral Pattern. Her main interest lies in acquiring information in a non-intrusive way, from the human's interaction with the computer, namely to assess attention and engagement. Dalila has authored of over 9 publications in peer-reviewed international journals, book chapters and conference proceedings.

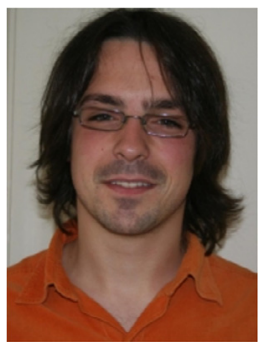

Davide Carneiro holds a Ph.D. with European Honors attributed under a joint Doctoral Programme in Computer Science that aggregates three top Portuguese Universities: Universidade do Minho, Universidade de Aveiro and Universidade do Porto. He is currently an Invited Professor at the Polytechnic Institute of Porto. For two years he was also an Invited Professor at the Department of Informatics of the University of Minho.

He develops scientific research in the fields of Artificial Intelligence and Machine Learning, with applications in Human-Computer Interaction and Context-aware Computing. Specifically, in the last years he has been working in extracting information from the human's interaction with computers and mobile devices, namely to assess stress and mental fatigue, building models of how individuals behave in different contexts and under different states. 


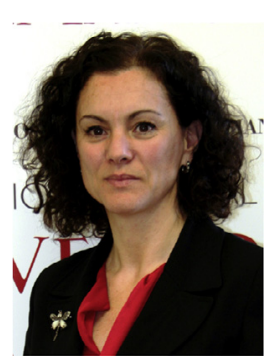

Amparo Jiménez Vivas is Full Professor in Educative and Professional Diagnosis at the Pontifical University of Salamanca, Spain. She holds a Ph.D. in Education and Psicology and her research interests are focused on the diagnosis of educational needs in different environments. She was head of the General Foundation of the Pontifical University of Salamanca from 2012 to 2015. She has published several paper in recognized conferences and Journals.

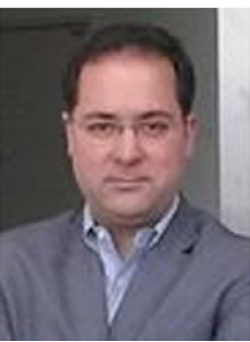

Paulo Novais is an Associate Professor with Habilitation of Computer Science at the Department of Informatics, in the School of Engineering of the University of Minho (Portugal) and a researcher at the ALGORITMI Centre in which he is the coordinator of the research group ISLab - Synthetic Intelligence. He is the director of the Ph.D. Program in Informatics and co-founder and Deputy Director of the Master in Law and Informatics at the University of Minho.

His main research aim is to make systems a little more smart, intelligent and also reliable. He is the co-author of over 230 book chapters, journal papers, conference and workshop papers and books.

He is the president of APPIA (the Portuguese Association for Artificial Intelligence) for 2016/2017, Portuguese representative at the IFIP (International Federation for Information Processing) - TC 12 - Artificial Intelligence, namely chair of the Working Group on Intelligent Agent (WG12.3) and member of the Artificial Intelligence Applications (WG12.5), and member of the executive committee of the IBERAMIA (IberoAmerican Society of Artificial Intelligence). 